\title{
Implementation via Rights Structures with Minimal State Spaces*
}

\author{
Semih Koray** \\ Bilkent University
}

\author{
Kemal Yıldız*** \\ Bilkent University
}

\begin{abstract}
In a recent paper, Koray and Yild1z (2018) propose implementation via rights structures ( $\Gamma$-implementability), which offers a simple framework for implementation formulated in a language closer to real-life mechanisms. They show that canonical rights structures, which renders a natural interpretation, are sufficient for implementing any image monotonic rule. However, one can question the simplicity of the rights structure on the basis of its number of states. In this paper, we address this question by formulating a rights structure which implements any $\Gamma$-implementable social choice rule with a minimal state space among all individual-based rights structures.

Keywords: Implementation, rights structures, Nash equilibrium, monotonicity, social choice rule, critical profile.

JEL Classification: D01.
\end{abstract}

\section{Minimal Durum Uzayı ile Haklar Yapısı Aracılığıyla Uygulanabilirlik}

\section{Özet}

Yakın zamanlı makalelerinde Koray ve Yıldız (2018) uygulama problemi için, gerçek hayattaki mekanizmalara benzer bir dilde ifade edilen, basit bir çerçeve öne sürmüşlerdir. Bu çalışmalarında tabi bir yorumlamaya müsade eden bir haklar yapısı sınıfının tüm görüntü tekdüze olan kuralları uygulamak için yeterli olduğunu göstermişlerdir. Ancak, bir haklar yapısının basitliğinin, tasarımında kullanılan durumların sayısına bağlı olabileceği öne sürülebilir. Bu makalede herhangi $\Gamma$-uygulanabilir bir sosyal seçme kuralını, tüm birey temelli haklar yapısı arasından, en az sayıda durumu kullanarak uygulama problemini ele almaktayı.

Anahtar Kelimeler: Uygulama, haklar yapısl, Nash dengesi, tekdüzelik, sosyal seçme kural, kritik profil. JEL Sinıflandirmast: D01.

* This paper subsumes and extends Section 1.6 of Y1ld1z (2013).

** Semih Koray. Bilkent University, Department of Economics. Email: ksemih@ bilkent.edu.tr

*** Kemal Y1ldız. Bilkent University, Department of Economics. Email: kemal.yildiz@ bilkent.edu.tr 


\section{Introduction}

Several institutional real-life mechanisms such as constitutions, legal codes, unwritten social norms and conventions aim to rule out unacceptable outcomes and implement only the socially acceptable ones under different circumstances. In implementation theory, we search for game forms to implement these socially acceptable outcomes by adhering to widely-used notions of equilibrium. However, our view is that the resulting game forms are quite different from the institutional mechanisms that we observe. To address this problem Y1ld1z (2013) and Koray and Yildiz (2018) propose and analyze a framework for implementation which is formulated in a language closer to these real-life mechanisms.

Consider any setting where it is given that, at each preference profile of a given society, some alternatives are in some sense socially acceptable and the rest are not. Implementation theory addresses the following question: Can we structure the interaction among individuals so that this interaction results in socially acceptable outcomes at each preference profile of the society? In the existing literature that answers this question, game forms are designed to implement socially acceptable outcomes by adhering to widely-used notions of equilibrium in different informational settings. A persistent criticism of the theory is that the game forms used in the general proofs have unnatural features that detract from the relevance of the theory. These unnatural features are "integer games" or "modulo games" that turn out to be an integral part of these constructions. These constructions are used to eliminate strategies with unacceptable outcomes from the equlibria ${ }^{1}$. To the best of our knowledge, the mechanisms used in the literature without these unnatural features work only in very particular settings.

Koray and Y1ldiz (2018) introduce a new societal framework for implementation which takes the power distribution in the society, represented by a rights structure, as its point of departure. A "rights structure" as introduced by Sertel (2001), roughly specifies the power of each coalition to block certain outcomes from being selected, in favor of another outcome. In the course of a presidential election, for example, every voter has the right to vote for a candidate or to abstain. On the other hand, blocking the election of a candidate as the president would typically require a majority of the voters. As another example, consider an institution consulting with a group of experts to undertake a project from among several options. The institution may eliminate one project in favor of another, if a consultant comes up with evidence, related to his area of expertise, that supports the other project.

More formally, a rights structure $\Gamma$ is a triple $(S, h, \gamma)$ and is the object of design. A non-empty set $S$ denotes a collection of (social) states that reflects the set of all possible situations that the society may end up with, possibly supported by any argument or evidence. A state can represent a resource allocation, an economic, political or legal arrangement. Furthermore, a state can itself be a constitution or legal code. An outcome function $h$ maps each state to an alternative. The code of rights $\gamma$ associates each ordered distinct pair $(s, t)$ of states, with a family of coalitions, $s \stackrel{\gamma}{\rightarrow} t$, which are entitled to approve the change from state $s$ to state $t$. Given a preference profile of the society, $u$, a coalition benefits from a change of state $s$ to state $t$ if all the members of this coalition prefer $h(t)$ to $h(s)$. The set of those coalitions that benefits from a change of state $s$ to

\footnotetext{
${ }^{1}$ For a more detailed discussion of why these constructions are problematic one can consult [Jackson (1992)].
} 
state $t$ is denoted by $s \stackrel{u}{\rightarrow} t$.

In this setting, the existence of a coalition endowed with the right to approve the change from state $s$ into another state $t$, conjoined with its willingness to do so means that $s$ cannot be an equilibrium state. Put differently, given a rights structure $\Gamma=(S, h, \gamma)$, a state $s$ is an equilibrium of this rights structure at preference profile $u$, if for each other state $t$, there is no coalition $K$ (i) which is entitled to approve a change from $s$ to $t$ and (ii) each member of $K$ prefers $h(t)$ to $h(s)$, i.e. $(s \stackrel{\gamma}{\rightarrow} t) \cap(s \stackrel{u}{\rightarrow} t)=\emptyset$.

Given a society and a set of alternatives, a social choice rule $F$ yields the acceptable alternatives at each preference profile. A social choice rule $F$ is implementable via the rights structure $\Gamma=$ $(S, h, \gamma)$, if at each preference profile, the set of alternatives chosen by $F$ coincides with the equilibrium outcomes of the rights structure at that preference profile. Koray and Y1ld1z (2018) show that $\Gamma$-implementable social choice rules are characterized by a slight strengthening of Maskin monotonicity, called image monotonicity. They observe that any $\Gamma$-implementable rule can be implemented in an individual-based manner, where if a coalition has the right to approve a change of state from $s$ to $t$, then this coalition must be a singleton. In other words, for each pair of distinct states $s$ and $t, s \stackrel{\gamma}{\rightarrow} t$ is either empty or consists of single agents.

Implementation via rights structures proposes a simple environment for implementation. Along these lines, Koray and Y1ldiz (2018) show that canonical rights structures, which have a natural interpretation, are sufficient for $\Gamma$-implementing any image monotonic rule. However, one can question the simplicity of the rights structure on the basis of its number of states. In this paper, we address this question by formulating a rights structure which implements any $\Gamma$ - implementable social choice rule with a minimal state space among all individual-based rights structures. Reichelstein and Reiter (1988) and McKelvey (1989) pose similar questions in the context of classical implementation via game forms.

In the rest of the paper, we first introduce the basics of $\Gamma$-implementation, and provide examples of $\Gamma$-implementable social choice rules. Then, we report a key relevant result from Koray and Y1ldiz (2018). Finally, we pose the question of implementability with a minimal state space and provide the answer in Proposition 1, which is the main result of this paper.

\section{Model}

We use $A$ to denote the non-empty, finite alternative set, and $N$ to denote a non-empty, finite set of $n$ agents. Each non-empty subset of $N$ is called a coalition and denoted generically by $K$.

For given $A$ and $N$, for each $i \in N, u_{i}$ denotes the preference relation ${ }^{2}$ of agent $i$. For each pair $a, b \in A$ with $a \neq b, a u_{i} b$ denotes $i$ prefers $a$ to $b$. A preference profile and the collection of all preference profiles are respectively denoted by $u=\left[u_{1}, \ldots, u_{n}\right]$ and $\mathcal{P}$. A social choice rule (SCR) $F$ maps each preference profile into a subset of $A$, i.e. $F: \mathcal{P} \rightarrow 2^{A}$. Notice that we allow the $\mathrm{SCR} F$ to be possibly empty valued. However, this specification does not play any role for the

\footnotetext{
${ }^{2}$ A preference relation is a complete, transitive, antisymmetric binary relation on $A$.
} 
general results that we obtain, but only provides ease of exposition for some of the examples we consider.

In classical implementation, the design object is a mechanism which is a pair $(M, g) . M=\prod_{i \in N} M_{i}$ denotes the joint strategy space, where $M_{i}$ stands for the strategy set of agent $i$ and $g$ is the outcome function which maps every joint strategy to an alternative, i.e. $g: M \rightarrow A$. A mechanism $(M, g)$ combined with a preference profile $u \in \mathcal{P}$, constitutes a normal form game. The set of pure strategy Nash equilibria of this game is denoted by NE $(M, g, u)$. We say a social choice rule $F$ is Nash implementable via a mechanism $(M, g)$, if at each preference profile $u$, alternatives chosen by $F$ coincide with the Nash equilibrium outcomes of the game at given $u$, i.e. for each $u \in \mathcal{P}$, we require $F(u)=\{g(s): s \in N E(M, g, u)\}$.

In Koray and Y1ld1z (2018)'s framework a rights structure, which was first introduced by Sertel (2001), is the design object. A rights structure $\Gamma$ is a triple $(S, h, \gamma)$. We use $S$ to denote the state space and $h$ to denote the outcome function which maps each state to an alternative, i.e. $h: S \rightarrow A$. Let $S \times S$ stand for the set of all ordered pairs $(s, t)$ with $s \neq t$.

Given a state space $S$, a code of rights specifies for each pair $(s, t) \in S \times S$ a family of coalitions denoted by $s \stackrel{\gamma}{\rightarrow} t$. We interpret that each coalition in $s \stackrel{\gamma}{\rightarrow} t$ is entitled to approve a change from $s$ to $t$, by the code of rights $\gamma$. Given a pair $(S, h)$ and a preference profile $u$, for each $(s, t) \in S \times$ $S$, a coalition $K$ prefers $t$ to $s$, denoted by $K \in s \stackrel{u}{\rightarrow} t$, if and only if for each $i \in K, h(t) u_{i} h(s)$. In order to define $\Gamma$-implementability, first we will specify the $\Gamma$-equilibrium notion which plays the role of solution concepts (e.g. Nash) in classical implementation.

Definition 1 Given a rights structure $\Gamma=(S, h, \gamma)$, for each $u \in \mathcal{P}$, we say $s \in S$ is a $\Gamma$-equilibrium at $u$ iffor each $t \in S$

$$
(s \stackrel{\gamma}{\rightarrow} t) \quad \cap \quad(s \stackrel{u}{\rightarrow} t)=\varnothing
$$

In other words, a state $s$ is a $\Gamma$-equilibrium at preference profile $u$, if there is no other state $t$ and a coalition $K$ (i) which is entitled to approve a change from $s$ to $t$ and (ii) each member of $K$ prefers $h(t)$ to $h(s)$. We denote the $\Gamma$-equilibria set at preference profile $u$ by $E(\Gamma, u)$.

Definition 2 A SCR $F$ is $\Gamma$-implementable if there exits a rights structure, $\Gamma=(S, h, \gamma)$, such that for each $u \in \mathcal{P}, F(u)=\{h(s): s \in E(\Gamma, u)\}$.

Being similar to Nash implementability, a social choice rule $F$ is implementable via the rights structure $\Gamma$, if at each preference profile $u$, alternatives chosen by $F$ coincide with the outcomes of the $\Gamma$-equilibrium at $u$, denoted by $h(E(\Gamma, u))$. To further motivate the analysis to follow, we will present some $\Gamma$-implementable rules and the rights structures that implement these rules.

\section{Examples}

Except for the majority rule example (Example 5), these examples are different from the ones reported by Koray and Yildiz (2018). We revisit the majority rule example of Koray and Yildiz to 
illustrate the restriction imposed by implementation via minimal rights structures.

Example 1 (Pareto Rule) For each society $N$, alternative set $A$ and a given preference profile $u, x \in F(u)$ if and only if there is no other alternative $y$, which Pareto dominates $x^{3}$. To see that $F$ is $\Gamma$-implementable, let us choose $S$ as the set of alternatives and the outcome function as the identity map. Let the code of rights be such that only the entire society, $N$, has the right to make a movement among any two states. One can easily verify that $F$ is implementable via this rights structure.

Example 2 (Condorcet Rule) For each society $N$ with at least three agents, alternative set $A$ and a given preference profile $u, F(u)=x$ if and only iffor any other alternative $y$, at least two of the agents prefer $x$ to $y$. To see that $F$ is $\Gamma$-implementable, let us choose $S$ as the set of alternatives and the outcome function as the identity map. Let the code of rights be such that, any coalition consisting of any two agents has the right to make a movement among any two states. One can easily verify that $F$ is implementable via this rights structure.

Example 3 (Guilty vs. Innocent) Consider a set of agents (e.g. jurors) $N=\{1,2, \ldots, n\}$ who will decide whether a suspect is guilty $(G)$ or innocent $(I)$. Suppose they decide that he is $I$ if and only if at least $k$ agents think he is so. Put differently, if at least $k$ jurors prefer I to $G$.

To see that for each $k \in\{1,2, \ldots, n\}$, this rule is $\Gamma$-implementable, first consider the preference profiles of the form $u_{I}$ where exactly $k$ agents prefer $I$ to $G$ and the rest prefer $G$ to $I$ and also consider the preference profiles of the form $u_{G}$ where exactly $n-k+1$ agents prefer $G$ to $I$ and the rest prefer $I$ to $G$. Let $S$ consist of the preferences of the form $u_{I}$ and of the form $u_{G}$ with the outcome function $h$ mapping a state of the $u_{I}$ form to $I$ and $u_{G}$ form to $G$. Let the code $\gamma$ of rights entitle any agent $i$ to approve a change from a state of the form $u_{I}$ to a state of the form $u_{G}$ if and only if $i$ prefers $I$ to $G$ at the particular state of the form $u_{I}$, so $i$ must be among the $k$ agents who prefers $I$ to $G$ at that particular $u_{I}$. Similarly, let $\gamma$ entitle any agent $j$ to move from a state of the form $u_{G}$ to a state of the form $u_{I}$ if and only if $j$ prefers $G$ to $I$ at the particular state of the form $u_{G}$, so $j$ must be among the $n-k+1$ agents who prefer $G$ to $I$ at that particular $u_{G}$.

For given $(S, h)$ let us see that $\gamma$ implements $F$. Let $u^{*}$ be the true preference profile. Suppose $F\left(u^{*}\right)=I$. Now, choose any $k$ agents who prefer $I$ to $G$ at $u^{*}$ and consider a new preference profile where all the rest prefer $G$ to $I$. Let this new profile be $u^{\prime}$. Notice that $u^{\prime}$ is of the form $u_{I}$, so we have $u^{\prime} \in S$ and for each state $v^{\prime}$ of the form $u_{G}$ and $i \in N$, we have $\{i\} \in u^{\prime} \stackrel{\gamma}{\rightarrow} v^{\prime}$ only if $I u_{i}^{\prime} G$. Hence, it is also true that $I u_{i}^{*} G$ and we obtain $u^{\prime} \in E\left(\Gamma, u^{*}\right)$. On the other hand, consider any state $v^{\prime}$ of the form $v$. Since there are at least $k$ agents who prefer $I$ to $G$ at $u^{*}$, there exists $i \in N$ with $\{i\} \in v^{\prime} \stackrel{\gamma}{\rightarrow} u^{\prime}$ and $I u_{i}^{*} G$, so $v^{\prime} \notin E\left(\Gamma, u^{*}\right)$. Thus we can conclude that $F\left(u^{*}\right)=$ $h\left(E\left(\Gamma, u^{*}\right)\right)$. Symmetric arguments work if $F\left(u^{*}\right)=G$.

Example 4 Let $N=\{1,2,3\}$ and $A=\{a, b, c\}$. Suppose for each preference profile $u$, and alternative $x \in A \backslash\{a\}, x$ is chosen if and only if $x$ is the Condorcet winner, and a is chosen if and only if $a$ is the Condorcet winner with the additional requirement that for every $i \in N, a$ is

\footnotetext{
${ }^{3}$ That is, for each $i \in N, y u_{i} x$.
} 
preferred to $c$. This choice rule explicitly suggests the code of rights where each coalition with at least two agents belongs to $x \stackrel{\gamma}{\rightarrow} y$ for each $x, y \in A$ and additionally any agent individually belongs to $a \stackrel{\gamma}{\rightarrow}$ c. It is easy to verify that $\gamma$ implements this choice rule which is not Nash-implementable.

Example 5 ( Majority Rule) Let $N=\{1,2,3\}$ and $A=\{a, b\}$. For each preference profile $u$, $F(u)=a$ if and only if $a$ is preferred to $b$ by at least two agents. Notice that there are four profiles $\left\{u^{1}, u^{2}, u^{3}, u^{4}\right\}$ where $a$ is chosen by $F$ since $a$ is preferred to $b$ by $N,\{1,2\},\{1,3\}$, and $\{2,3\}$ respectively. Similarly let $\left\{v^{1}, v^{2}, v^{3}, v^{4}\right\}$ be the profiles where the roles of $a$ and $b$ are changed, so $b$ is chosen by $F$.

Consider the following rights structure $\Gamma^{d}=\left(S^{d}, h^{d}, \gamma^{d}\right)$ tailored for this rule. Namely, we have $S^{d}=\left\{\left(a, u^{m}\right),\left(b, v^{m}\right)\right\}_{m=1}^{4}$, where outcome function $h^{d}$ maps each $\left(a, u^{m}\right)$ to $a$ and each $\left(b, v^{m}\right)$ to $b$. Now, the code $\gamma^{d}$ of rights entitles any coalition $K$ to approve a change from any state $\left(a, u^{m}\right)$ to another $\left(b, v^{r}\right)$ if and only if for some $i \in K, a u_{i}^{m} b$.

To see that $\Gamma^{d}$ implements $F$, suppose the true preference profile is of the form $u^{m}$, so we have $F\left(u^{m}\right)=a$. Now, consider the state $\left(a, u^{m}\right)$. By the design of $\gamma^{d}$, there can be no $i \in N$ with $i \in$ $\left(a, u^{m}\right) \stackrel{\gamma^{F}}{\rightarrow}\left(b, v^{r}\right)$ and $b u_{i}^{m} a$, it follows that $\left(a, u^{m}\right) \in E\left(\Gamma^{F}, u^{m}\right)$. On the other hand for each $\left(b, v^{r}\right)$ since at least two agents prefer $b$ to $a$ at $v^{r}$ and $a$ to $b$ at $u^{m}$, there necessarily exists $i \in N$ such that $b v_{i}^{r} a$ and $a u_{i}^{m} b$. By the design of $\gamma^{d}$, this means $i \in\left(b, v^{r}\right) \stackrel{\gamma^{F}}{\rightarrow}\left(a, u^{m}\right)$ and $a u_{i}^{m} b$. Thus $\left(b, v^{r}\right) \notin E\left(\Gamma^{F}, u^{m}\right)$ and we obtain $h^{d}\left(E\left(\Gamma^{F}, u^{m}\right)\right)=a$. For a true preference profile of the form $v^{m}$ symmetric arguments work.

\section{Monotonicity and $\Gamma$-implementation}

In classical implementation theory, Maskin (1985) shows that any Nash implementable SCR is monotonic, and monotonicity combined with no veto power condition is sufficient for Nash implementability in cases involving at least three agents. Let us remember this well-known monotonicity condition for implementation. A social choice rule $F$ is monotonic, if for each $u^{1}, u^{2} \in \mathcal{P}$ and $a \in F\left(u^{1}\right)$, we have $a \in F\left(u^{2}\right)$ whenever for every $i \in N$ and $b \in A$, if $a u_{i}^{1} b$ then $a u_{i}^{2} b$ holds.

Koray and Yildiz (2018) propose a monotonicity condition that is slightly stronger than that of Maskin (1985). They name this condition image monotonicity, and show that a SCR, $F$ is $\Gamma$ implementable if and only if $F$ is image monotonic. Moreover, it follows from the proof of this proposition that any $\Gamma$-implementable rule can be implemented via an individual-based (IB) rights structure where for each distinct state pair $(s, t)$, either some single agents have the right to move from $s$ to $t$, or no coalition has the right for such a movement.

Before proceeding to the definition of image monotonicity, let us introduce some useful notation. Let $I(F)$ denote the image of $F$; i.e $I(F)=\{a \in A: a \in F(u)$ forsome $u \in \mathcal{P}\}$. The lower contour set of $u_{i}$ with respect to alternative $a \in A$, denoted by $\mathrm{L}\left(u_{i}, a\right)$, is the set of alternatives to which $a$ is preferred by agent $i$, i.e. $\mathrm{L}\left(u_{i}, a\right)=\left\{b \in A: a u_{i} b\right\}$. By using this definition, 
(Maskin) monotonicity can be restated as follows: A social choice rule $F$ is monotonic, if for each $u^{1}, u^{2} \in \mathcal{P}$ and $a \in F\left(u^{1}\right)$, we have $a \in F\left(u^{2}\right)$ whenever for every $i \in N, \mathrm{~L}\left(u_{i}^{1}, a\right) \subseteq$ $\mathrm{L}\left(u_{i}^{2}, a\right)$.

Image monotonicity: A social choice rule $F$ is image monotonic, if for each $u^{1}, u^{2} \in \mathcal{P}$, and each $a \in F\left(u^{1}\right)$, we have $a \in F\left(u^{2}\right)$ whenever for every $i \in N$,

$$
\mathrm{L}\left(u_{i}^{1}, a\right) \cap I(F) \subseteq \mathrm{L}\left(u_{i}^{2}, a\right) .
$$

Theorem 1 (Koray and Yldız (2018)) Given a social choice rule $F$, the following are equivalent, i. $F$ is image monotonic

ii. $F$ is $\Gamma$-implementable

iii. $F$ is $\Gamma$-implementable via an IB rights structure.

\section{$\Gamma$-implementation with minimal state spaces}

A natural measure for the simplicity of a rights structure is its number of states. For example, let us turn back to the example of a simple majority rule. Recall that $u^{1}$ and $v^{1}$ were standing for the profiles where every agent top ranks $a$ and every agent top ranks $b$ respectively. Now let us remove $u^{1}$ and $v^{1}$ from the state space $S$ and keep $h$ and $\gamma$ as they are on $S \backslash\left\{u^{1}, v^{1}\right\}$. It is easy to notice that if the true profile is say $u^{1}$, then $E\left(\Gamma, u^{1}\right)=\left\{u^{2}, u^{3}, u^{4}\right\}$, so we obtain that this new rights structure implements the simple majority rule as well. However, apparently there are six states in this rights structure versus eight in the original one. This brings the question of whether one can describe a class of rights structures which would implement an image monotonic rule with the minimum possible number of states.

Next, we introduce the critical rights structures and show that if a social choice rule is implementable via a rights structure then the state space of this rights structure should be at least as large as that of the critical rights structure associated with the given social choice rule .

\section{Critical rights structures}

For a given SCR $F$, a critical rights structure $\Gamma^{c}=\left(S^{c}, h^{c}, \gamma^{c}\right)$. The state space, $S^{c}$, consists of alternative and critical preference profile pairs $(a, u)$. First, we formally define the critical profiles.

For each $a \in A$, let us first define a binary relation $\succeq_{a}$ over the set of preference profiles. For each $u, v \in \mathcal{P}$, we say $v \geq_{a} u$ if and only if $v$ is an a-monotonic transformation of $u$. Namely, if for each $i \in N$, we have $\mathrm{L}\left(u_{i}, a\right) \subseteq \mathrm{L}\left(v_{i}, a\right)$. We say a preference profile $u$ is critical for $a$ if $u$ is minimal with respect to $\geq_{a}$, i.e. there is no preference profile $v$ such that $u \geq_{a} v .{ }^{4}$

For each $u, u^{\prime}$ that are $a$-critical, if for each $i \in N, \mathrm{~L}\left(u_{i}, a\right)=\mathrm{L}\left(u_{i}^{\prime}, a\right)$, then $u$ is $a$-equivalent to $u^{\prime}$. For each $a$-critical profile $u$, we denote the associated equivalence class by $[u]$. Let $\Lambda(F, a)$ stand for a set of a-critical profiles such that for each equivalence class $[u]$,

\footnotetext{
${ }^{4} \mathrm{An}$ equivalent description is as follows. For each $a \in A$, we say a preference profile $u$ is critical for $a$, if any agent $i$ reverses his preference between $a$ and $b$ for any $b \in L\left(u_{i}, a\right)$, then $F$ no longer chooses $a$ in the new profile.
} 
$\Lambda(F, a)$ contains a unique representative a-critical profile $u \in[u]$.

Remark 1 Since $F$ is image monotonic, for each $a \in I(F)$ we have $\Lambda(F, a) \neq \emptyset$. Since a $\in$ $I(F)$, there exists a preference profile $u$, such that $a \in F(u)$. If $u^{\prime}$ is an a-monotonic transformation of $u$, then it follows from image monotonicity that $a \in F\left(u^{\prime}\right)$ as well. Since $A$ is a finite set there exists $v \in \mathcal{P}$ such that $v$ is minimal with respect to $\succeq_{a}$ and for each $u^{\prime}$ which is an a-monotonic transformation of $u$ we have $u^{\prime} \geq_{a} v$.

Now, for a given image monotonic social choice rule $F$, we define the critical state space $S^{c}$ as

$$
S^{c}=\{(a, u): \quad u \quad \in \Lambda(F, a)\}
$$

Let $h^{c}$ and $\gamma^{c}$ be such that $h^{c}$ maps each $(a, u) \in S^{c}$ to alternative $a$, and the code of rights, $\gamma^{c}$, entitle each coalition $K$ to approve a change from any state $(a, u)$ to another state $(b, v)$ if and only if there exists $i \in K$ such that $a u_{i} b$.

Proposition 1 If an individual-based rights structure, $\Gamma=(S, h, \gamma)$, implements $F$, then the number of states in $S$ is at least equal to the number of states in $S^{c}$, i.e. $|S| \geq\left|S^{c}\right|$.

Proof. Let $F$ be a $\Gamma$-implementable social choice rule.

Step 1: We will show that $F$ is implementable via $\Gamma^{c}$.

It follows from Proposition 1 of Koray ve Yildiz (2018) that $F$ is implementable via the the canonical rights structure $\Gamma^{F}=\left(S^{F}, h^{F}, \gamma^{F}\right)$ designed as follows. The state space $S^{F}$ is defined as the graph of $F$, i.e. $S^{F}=\{(a, u) \in A \times \mathcal{P}: \quad a \in F(u)\}$. The outcome function $h^{F}$ maps each $(a, u) \in S^{F}$ to $a$. The code of rights $\gamma^{F}$ will entitle an agent $i$ to move from state $(a, u)$ to another state $(b, v)$ if and only if $i$ prefers $a$ to $b$ at $u$, i.e. $a u_{i} b$.

By definition, $S^{c} \subseteq S^{F}$ and $h^{c}$ coincides with $h^{F}$ over $S^{c}$. Moreover, $\gamma^{c}$ coincides with $\gamma^{F}$, over $S^{c} \times S^{c}$. We will show that for each $u \in \mathcal{P}, h^{F}\left(E\left(\Gamma^{F}, u\right)\right)=h^{c}\left(E\left(\Gamma^{c}, u\right)\right)$. Let $(a, u) \in$ $E\left(\Gamma^{F}, u\right)$. This means $a \in F(u)$, so there exists an $a$-critical profile $u^{\prime}$, such that $u \geq_{a} u^{\prime}$. We will show that $\left(a, u^{\prime}\right) \in E\left(\Gamma^{c}, u\right)$. Suppose not, i.e. there exists $(b, v) \in S^{c}$, and $i \in N$ where $\{i\} \in\left(a, u^{\prime}\right) \stackrel{\gamma}{\rightarrow}(b, v)$ and $b u_{i} a$. By the design of $\gamma^{c}$, we can have $\{i\} \in\left(a, u^{\prime}\right) \stackrel{\gamma^{c}}{\rightarrow}(b, v)$ only if $a u_{i}^{\prime} b$. Since $u \succeq_{a} u^{\prime}$, it follows $a u_{i} b$ as well. But this contradicts $(a, u) \in E\left(\Gamma^{F}, u\right)$.

For the other direction, let $\left(a, u^{\prime \prime}\right) \in E\left(\Gamma^{c}, u\right)$. Notice that it is not necessarily true that $u \geq_{a} u^{\prime \prime}$. Next, we will show that $\left(a, u^{\prime \prime}\right) \in E\left(\Gamma^{F}, u\right)$. Suppose not, i.e. there exists $(b, v) \in S^{F}$, and $i \in N$ such that $\{i\} \in\left(a, u^{\prime \prime}\right) \stackrel{\gamma^{F}}{\rightarrow}(b, v)$ and $b u_{i} a$. By the design of $\gamma^{d}$, we can have $\{i\} \in$ $\left(a, u^{\prime \prime}\right) \stackrel{\gamma^{F}}{\rightarrow}(b, v)$ only if $a u^{\prime \prime}{ }_{i} b$. Moreover, $(b, v) \in S^{d}$ implies $b \in F(v)$, so there exists a $b$ - critical profile $v^{\prime}$ with $v \geq_{b} v^{\prime}$. Hence we have $\left(b . v^{\prime}\right) \in S^{c}$. But now, $\{i\} \in$ $\left(a, u^{\prime \prime}\right) \stackrel{\gamma^{c}}{\rightarrow}\left(b, v^{\prime}\right)$ and $b u_{i} a$. This contradicts $\left(a, u^{\prime \prime}\right) \in E\left(\Gamma^{c}, u\right)$.

Step 2: Let $\Gamma=(S, h, \gamma)$ be any rights structure which implements $F$. We will show that $|S| \geq$ $\left|S^{c}\right|$.

It is clear that $|S| \geq|I(F)|$. Moreover, for each distinct $(a, u),\left(a, u^{\prime}\right) \in S^{c}$ there exist $s, s^{\prime} \in S$ 
such that $s \in E(\Gamma, u)$ and $s^{\prime} \in E\left(\Gamma, u^{\prime}\right)$, with $h(s)=h\left(s^{\prime}\right)=a$. Next, we will show that indeed $s$ and $s^{\prime}$ are distinct. Suppose not, i.e. $s=s^{\prime}$. Since $u \neq u^{\prime}$, this means there exists $i \in N$ and $b \in A \backslash\{a\}$ such that w.l.o.g. $a u_{i} b$ and $b u_{i}^{\prime} a$. Consider the profile $v$ which is a copy of $u$ with the exception that we have $b v_{i} a$. Since $u$ is $a$-critical, we have $a \notin F(v)$. Since $F(v)=$ $E(\Gamma, v)$, where $\gamma$ is individual-based, this is possible only if we have $\{i\} \in s \stackrel{\gamma}{\rightarrow} t$ for some $t \in S$ with $h(t)=b$. But, since we also have $h(s) u_{i}^{\prime} h(t)$, this contradicts $s \in E\left(\Gamma, u^{\prime}\right)$. So far, we have shown that for each $a \in A$ and distinct $(a, u),\left(a, u^{\prime}\right) \in S^{c}$, there exist distinct $s, s^{\prime} \in S$ with $h(s)=h\left(s^{\prime}\right)=a$, it follows that $|S| \geq\left|S^{c}\right|$.

\section{Conclusion}

Koray and Yildiz (2018) recently proposed implementation via rights structures ( $\Gamma$ implementability), which takes a persistent criticism of implementation theory, demonstrating that the game forms used in the general constructions contain unnatural features such as an appended "integer game". Koray and Yıldız offer a simple framework for implementation formulated in a language closer to the real life mechanisms. They show that canonical rights structures, which render a natural interpretation, can be used to implement any image monotonic rule. However, one can also question the simplicity of a rights structure on the basis of its number of states. Here, as complementary to the analysis of Koray and Y1ld1z, we have shown that if a social choice rule is implementable via a rights structure then the state space of this rights structure should be at least as large as that of the critical rights structure associated with the given social choice rule . 


\section{References}

Jackson, Matthew O. (1992), "Implementation in undominated strategies: A look at bounded mechanisms." The Review of Economic Studies, 59 (4), 257-775.

Koray, Semih and Kemal Y1ldı (2018), “Implementation via rights structures.” Journal of Economic Theory, 176, 479-502.

Maskin, Eric (1985), “The theory of implementation in Nash equilibrium: A survey." In Social Goals and Social Organization (L. Hurwicz, D. Scheidler, and H. Sonnenschein, eds.), Cambridge University Press, 173-204.

McKelvey, Richard D. (1989), "Game forms for Nash implementation of general social choice correspondences.” Social Choice and Welfare, 6(2), 139-156.

Reichelstein, Stefan and Stanley Reiter (1988), "Game forms with minimal message spaces." Econometrica: Journal of the Econometric Society, 56 (3), 661-692.

Sertel, R. Murat (2001), "Designing rights: Invisible hand theorems, covering and membership." Mimeo, Boğaziçi University.

Y1ldı, Kemal (2013), Essays in Microeconomic Theory. Ph.D. thesis, New York University. 